\title{
Gamma-Rays from Grazing Incidence Cosmic Rays in the Earth's Atmosphere
}

\author{
Andrew Ulmer ${ }^{1}$ \\ Princeton University Observatory, Peyton Hall, Princeton, NJ 08544-1001, USA
}

Received —

Submitted to Astrophysical Journal Letters, Feb 5, 1994

${ }^{1}$ E-mail: andrew@astro.princeton.edu 


\begin{abstract}
Interactions of grazing incidence, ultra high energy cosmic rays with the earth's atmosphere may provide a new method of studying energetic cosmic rays with gamma-ray satellites. It is found that these cosmic ray interactions may produce gamma-rays on millisecond time scales which may be detectable by satellites. An extremely low gamma-ray background for transient gamma-ray events and a large area of interaction, the earth's surface, make the scheme plausible. The effective cross section of detection of interactions for cosmic rays above $10^{20} \mathrm{eV}$ is found to be more than two orders of magnitude higher than earth based detection techniques. This method may eventually offer an efficient way of probing this region of the cosmic ray energy spectrum where events are scarce. In this paper, a conceptual model is presented for the production of short bursts of gamma-rays based on these grazing incidence encounters with the earth's atmosphere.
\end{abstract}

Subject headings: atmospheric effects - cosmic rays - Earth - gamma-rays: bursts

Knowledge of the cosmic ray spectrum above $10^{20} \mathrm{eV}$ would provide important insight into both the acceleration and propagation of ultra high energy cosmic rays (UHE's). For instance, it has long been believed that there is a cutoff in the energy spectrum due to interactions between the cosmic rays and the microwave background (Greisen 1966; Zatsepin \& Kuzmin 1966). Despite recent progress (cf. Bird et al. 1994), and work over the last three decades, the study of this energy range remains limited by statistics and instrumental effects of earth based cascade detectors. For these reasons, it is of cardinal importance to consider alternative techniques to extend the study of this region. It is the purpose of this work is to investigate the feasibility of a detection method based on grazing incidence cosmic 
rays in the earth's atmosphere. A conceptual model of the gamma-ray production as well as some of the detectable characteristics are discussed. Specifically, energy requirements, frequency of occurrence, timescale, and energy spectrum are estimated. Parenthetically, this study was motivated by the announcement of the recent detection of what are believed to be short gamma-ray flashes from the direction of the earth by the Burst and Transient Source Experiment (BATSE) on the Compton Gamma Ray Observatory (CGRO) at the 1993 Huntsville Gamma-ray Burst Conference (Fishman et al. 1993), but no attempt is made here to make a detailed comparison with the reported phenomenon. It cannot yet be determined if there is a link between the grazing incidence cosmic rays and the detected flashes; however, UHE's are important regardless of their connection, or lack therof, to the BATSE events.

Cosmic rays are known to be incident on the earth at energies up to and above $10^{20} \mathrm{eV}$. Ultra high energy cosmic rays interact with the atmosphere causing a cascade of particles. Most observations of these cascades are ground based. However, it has been suggested that cosmic rays of $10^{19} \mathrm{eV}$ and above could be studied from space by observing optical ionization emission in the atmosphere (Benson \& Linsley 1979, Linsley 1979), although these methods were finally found to be impractical due primarily to the large optical background. Additionally, interactions on the solar surface of lower energy cosmic rays have previously been investigated theoretically and are suspected to produce a few $\sim 100 \mathrm{MeV}$ gamma-rays at a marginally detectable level (Seckel, Stanev, \& Gaisser 1991). Most relevant to the question of cosmic ray induced gamma-rays, is the earth gamma-ray background. This well-studied background is believed to be produced predominately by the bremsstrahlung of electrons from the cascades of cosmic rays (e.g. Beuerman 1971; Џmhof, Nakana, \& Reagan 1976; Dean et al. 1989). The detection method proposed here is based on intensity fluctuations in time of this background produced by the most energetic cosmic rays. 
The reason for interest in grazing as opposed to oblique incidence cosmic rays is the atmosphere is highly opaque to gamma-rays, with an attenuation depth of $\sim 15 \mathrm{gm} / \mathrm{cm}^{2}$ (e.g. Morris 1984). Cascades which produce gamma-rays deep in the atmosphere will not be seen by satellites. Gamma rays at an altitude of $\sim 30 \mathrm{~km}$ will travel one attenuation length, therefore, any atmospheric gamma-rays detectable from space are most likely to be generated in the stratosphere $(25-50 \mathrm{~km})$. In a typical cascade, the number of particles will increase while degrading in energy via strong interactions, bremsstrahlung, and pair production. When most of the constituents of the cascade have energies of $\sim 80 \mathrm{MeV}$, the cascade elements are primarily electrons, positrons, and photons. At this energy, the process reaches a maximum number of particles after which the electrons quickly lose their energy to ionization (cf. Sokolsky 1988, Longair 1992,Gaisser 1990). It takes UHE's about $800 \mathrm{gm} / \mathrm{cm}^{2}$ to reach this critical energy, whereas the column density at sea level is about $1000 \mathrm{gm} / \mathrm{cm}^{2}$. At maximum, the cascade is almost exclusively electrons and positrons and contains nearly all of the energy of the initial particle (Gaisser 1990). If the particles move tangent to the earth's surface and are high enough in the atmosphere so that the density is much smaller than at sea level, the entire cascade can have a path length of the order of hundreds of kilometers. If beamed similarly to downward moving particle cascades, the lower energy electrons are beamed into a cone of half-angle approximately $4^{\circ}$ (e.g. Bird et al. 1994). Additionally, at energies near the critical energy where the cascade has a maximum number of charged particles, the electrons will begin to spiral in the earth's magnetic field with radii of less than a kilometer which is much shorter than the path length. Although the radiation, which is primarily bremsstrahlung, from a single one of these electrons is beamed along the direction of motion, the gyration of the electrons will randomize the directions of motion and emission into a plane. If the incident cosmic ray is perpendicular to the magnetic field, the cascaded radiation will be beamed into approximately 0.9 sterradians corresponding to the angular area of a rotated cone of $4^{\circ}$. 
However, if the incident direction is parallel, the magnetic field will not have a large effect on the beaming area. For the calculations in the rest of the paper, an average beaming area of 0.5 sterradians is assumed. Planer randomization is not as important in the usual downward moving cascades detected from earth (e.g. Elbert, Stanev, \& Torii 1983) because the total path length of the subcritical energy particles is much shorter. If the cascade products are high enough in the atmosphere $\left(\sim 15 \mathrm{gm} / \mathrm{cm}^{2}\right)$, some of the gamma-ray photons will escape and may be detectable.

Currently, the most sensitive all-sky gamma-ray detector, BATSE is capable of detecting and directionally localizing a burst with about one hundred photons in the 50-300 keV range (Eishman et al. 1989). With this as a typical energy required for a detection, the total energy requirement in the initial particle can be estimated. If the radiation is beamed as discussed above and emitted from the stratosphere at a distance of 500 kilometers from the detector, then the total energy needed to detected by BATSE with an area of $\sim 1 \mathrm{~m}^{2}$ is estimated to be

$$
(0.5 \text { sterrad })(500 \mathrm{~km})^{2}(300 \mathrm{keV})(100 \text { photons }) / \epsilon_{-2} \approx 4 \times 10^{20} \mathrm{eV} / \epsilon_{-2},
$$

where $\epsilon_{-2}$ is taken as an efficiency normalized to 1 per cent. If the efficiency of converting energy into gamma-rays is approximately 1 per cent, then the method would examine extremely ultra high energy events, although in the future, larger, more sensitive experiments may be capable of observing the spectrum at lower energies. Although the efficiency should eventually be derived from extensive monte carlo simulations, it can be estimated by taking the ratio of the energy flux of the upward atmospheric gamma-ray background (Imhof, Nakana, \& Reagan 1976) to the energy flux of gamma-ray producing incident cosmic rays. Such estimates give values ranging from $\epsilon_{-2}=0.2-1$.

The integral flux of cosmic rays above $4 \times 10^{20} \mathrm{eV}$ is estimated to be $\sim 1.5 \times 10^{-20} \mathrm{~cm}^{-2} \mathrm{~s}^{-1}$ ster $\mathrm{rad}^{-1}$ by extending the spectrum from lower energies (e.g. Bird et al. 1994; 5okolsky 
1988). The area of the atmosphere observable by a satellite can be calculated to be approximately

$$
2 \pi r R \approx 1 \times 10^{7} \mathrm{~km}^{2}
$$

where $R$, the radius of the earth, is $6000 \mathrm{~km}$ and $r$, the height of a satellite orbit (taking GRO for example), is $400 \mathrm{~km}$. The fraction of acceptable incidence angles to the earth's surface can be estimated. The cascades travel many hundreds of kilometers in the atmosphere, and assuming it must reach maximum in about a 10 kilometer range of altitudes, incident angles to the horizon are required to be within a range of about $\pi / 100$. The second angular component is arbitrary so, the amount of acceptable incidence angles is $\sim 0.2$ sterradians Therefore, assuming this area of detection, acceptable incidence angles, and a beaming factor of individual events of $(0.5 / 4 \pi)$ the total number of detectable events in a one year period is found to be

$$
\frac{0.5}{4 \pi}\left(1.5 \times 10^{-20}\right)\left(1 \times 10^{7} \mathrm{~km}^{2}\right)\left(3 \times 10^{7} \mathrm{sec}\right)(0.2 \text { sterrad }) \approx 350 \text { events } .
$$

Because of the steep slope of the cosmic ray spectrum, the number of events can be increased/decreased by a factor of 100 for every decade decrease/increase of input cosmic ray energy above about $10^{16} \mathrm{eV}$. Consequently, the frequency of expected events scales strongly with the cosmic ray energy necessary for detection, and therefore $\epsilon_{-2}$.

The timescale of cosmic ray events are likely to be no more than a few milliseconds, because the light travel time across the earth is about 40 milliseconds, and only a fraction of the earth is observed by most satellites. This timescale is comparable to the timescales of a few milliseconds that have been observed by BATSE in the perturbation of arrival times of a short gamma-ray burst by reflection off the earth's atmosphere (Bhat et al. 1992). For relativistic particles, two milliseconds corresponds to distance scale of $600 \mathrm{~km}$. This distance is consistent with the region of atmosphere observed by a satellite in orbit at a height of $400 \mathrm{~km}$. Such a path length is plausible for a cascade in the atmosphere 
because the depth of shower maximum which occurs when the electrons reach the critical energy is nearly $1000 \mathrm{gm} / \mathrm{cm}^{2}$ for the most energetic UHE's, and the density at a height of 30 kilometers is $2 \times 10^{-5} \mathrm{~g} / \mathrm{cm}^{3}$ (U.S. Standard Atmosphere, 1976). Therefore, a cascade which lasts hundreds of $\mathrm{km}$ is possible.

Similar to the atmospheric background of gamma-rays, the UHE cascades may produce gamma-rays by bremsstrahlung or pair-annihilation (Beuerman 1971; Ling 1975). A rough estimate of the spectrum of grazing incidence UHE events is the background gamma-ray spectrum of the earth's atmosphere as observed by satellite. This background is believed to be primarily due to lower energy cosmic ray interactions and is characterized by a power law energy spectrum of $\mathrm{E}^{-1.4}$ counts $/ \mathrm{cm}^{2} / \mathrm{s} / \mathrm{keV}$ around $1 \mathrm{MeV}$ (Imhof, Nakana, \& Reagan 1976, Dean et al. 1989).

In conclusion, it appears that it is within the capabilities of current or next generation gamma-ray experiments to search for grazing incidence ultra high energy cosmic rays as short bursts of gamma-rays in the earth's atmosphere. One possible signature of UHE interactions is that, because some secondary products of the UHE may not degrade immediately, a few different maxima, or gamma-ray emission locations, may be produced which would differ by a distance of hundreds of kilometers. In these cases, the bursts may not appear as point sources to BATSE which may be able to localize the directions to better than 100 kilometers although the localization of bursts is a strong function of the incident spectrum and may be worse for spectra which differ from those of classical gamma-ray bursts for which the experiment was optimized (Brock et al. 1993; Horack et al. 1993). However, if many events were detected, it may be possible to statistically argue that the events are not point sources. Additionally, a detailed spatial distribution function could show anisotropies due to the earth's magnetic field or column densities in the atmosphere. There are many geometries and possible interactions that Can conceivably produce a large 
variety of events. For this reason, quantitative monte carlo studies of these interactions would yield more exact predictions of what would be observed from space. These ultra high energy cosmic ray interactions may eventually reveal valuable information about UHEs. For example, contingent on the assumptions presented above, if BATSE has detected none of these UHE events in two years of operation, one can place an upper limit on the integral flux of cosmic rays above approximately $4 \times 10^{20} \mathrm{eV}$ of $\sim 2 \times 10^{-22} \mathrm{~cm}^{-2} \mathrm{~s}^{-1}$ ster $\mathrm{rad}^{-1}$.

It is a pleasure to thank B. Paczyński, M. Ulmer, G. Fishman, T. Stanev, and the anonymous referee for helpful comments. This project was supported by the NASA grant NAG5-1901. 


\section{REFERENCES}

Benson, R. \& Linsley, J. 1979, 17th International Cosmic Ray Conference, 8, 145

Beuerman, K.P. 1971, J. Geophys. Res., 76, 4291

Bhat, P.N., et al. 1992, Nature, 359, 217

Bird, D.J., et al. 1994, ApJ, 424, 491

Brock, J.M., Fishman, G.J., Meegan, C.A., Wilson, R.W., Pendleton, G.N., Stollberg, \& W.S., Paciesas, W.S. 1993, Proc. of the St. Louis Compton Gamma-ray Obs. Conference (eds. Friedlander, M., Gehrels, N., \& Macomb, D. J.), 714 (AIP Pub. No. 280, New York)

Dean, A., et al. 1989, A\&A, 219, 358

Elbert, J.W., Stanev, T., \& Torrii, S. 1983, 18th International Cosmic Ray Conference, 6, 227

Fishman, G.J., et al. 1989, Proc. Gamma Ray Observatory Science Workshop (ed. Johnson, W.N.), 3-47 (Greenbelt, MD: NASA/GSFC)

Fishman, G.J., et al. 1993, announcement at Huntsville GRB workshop

Gaisser, T.K. 1990, Cosmic Rays and Particle Physics, (Cambridge U. Press)

Greisen, K. 1966, Phys. Rev. D, 47, 1919

Horack, J.M., et al. 1993, Proc. of the St. Louis Compton Gamma-ray Obs. Conference (eds. Friedlander, M., Gehrels, N., \& Macomb, D. J.), 714 (AIP Pub. No. 280, New York) 
Imhof, W.L., Nakano, G.H.,\& Reagan, J.B., 1976, J. Geophys Res., 81, 2835

Ling, J.C. 1975, J. Geophys Res., 80, 3241

Linsley, J. 1985, 19th International Cosmic Ray Conference, 3, 438

Longair, M.S. 1992, High Energy Astrophysics, Vol. 1. (Cambridge U. Press)

Morris, D. 1984, High Energy Transients in Astrophysics, ed. Woosley, S.E., 665 (New York: AIP Press)

Seckel, D., Stanev, T., \& Gaisser, T.K. 1991, ApJ, 382, 652

Sokolsky, P. 1988, Introduction to Ultrahigh Energy Cosmic Ray Physics, (Addison-Wesley Pub Co, Inc.)

U.S. Standard Atmosphere 1976, Pub. NOAA-S/T76-1562. Washington D.C.: U.S. Government Printing Office

Zatsepin, G.T. \& Kuzmin, V.A. 1966, Soviet Phys.-JEPT Lett., 4, 78

This manuscript was prepared with the AAS LATEX macros v3.0. 